Original Contribution

\title{
RIB FRACTURES IN BLUNT CHEST TRAUMA - ASSOCIATED THORACIC INJURIES
}

\author{
Iv. Dimitrov*, Iv. Novakov, P. Bonev, A. Uchikov \\ Department of General and Thoracic Surgery, Medical University- Plovdiv, University Hospital \\ "St.George"- Plovdiv, Bulgaria
}

\begin{abstract}
PURPOSE. The aim of our retrospective study was to analyze the patterns of associated thoracic injuries in patients underwent blunt chest trauma and rib fractures.

METHODS. The study included 212 patients with rib fractures due to blunt thoracic trauma. The mechanism of trauma, the type of rib fracture and the type of associated injuries were analyzed. RESULTS. The patients were divided in two groups according to the number of fractured ribs-group I included the patients with up to two fractured ribs (72 patients-33,9\%), and group II - with $\geq 3$ fractured ribs (140 patients-66,1\%). Associated chest injuries were present in 36 of the patients from group I (50\%), and in 133 patients from group II (95\%). Pulmonary contusion was the most common intrathoracicinjurie$65,6 \%$ of the cases. The mean hospital stay was 8,7 days. The lethality rate was $16,9 \%$-all of them due to the associated chest injuries.
\end{abstract}

CONCLUSIONS. The mortality related to rib fractures is affected by the associated thoracic injuries, the advanced age, and the number of fractured ribs.

Key words: blunt chest trauma, rib fractures, associated chest injuries, mortality

\section{INTRODUCTION}

Traumatic thoracic injury ranks second only to head injury as the leading cause of traumarelated death (1). Chest trauma accounts for $10 \%$ to $15 \%$ of trauma admissions and the rib fractures and the associated intrathoracic injuries in blunt chest trauma lead to a mortality of $15,5 \%$ to $25 \%(2,3,4)$. Blunt thorax traumas constitute the majority of thorax traumas and the rib fractures are their most common result $(5,6)$. The vehicle accidents continue to be the most common cause of trauma. The vast majority of the patients are under 65 years old, mostly male patients. Rib fractures are often underestimated, proper follow-up and treatment are not provided, which results in higher morbidity and mortality. However, the associated chest injuries in patients underwent blunt trauma and fracture of ribs, are much more important. Many studies show that in only $5 \%$ of the patients following blunt chest trauma, the fractured ribs are an isolated thoracic injury. In all of the other $95 \%$ of the cases, the rib fractures are associated with other intrathoracic injuries $(7,8,9)$.

\footnotetext{
*Correspondence to: Ivan N. Dimitrov Medical University - Plovdiv; Department of Special Surgery; e-mail: indimitrov@abv.bg
}

\section{OBJECTIVE}

The aim of our study was to report the data on 212 cases of blunt chest trauma and rib fractures and to present the type and the severity of the associated intrathoracic injuries.

\section{MATERIAL AND METHODS}

We retrospectively evaluated the data of 212 patients with rib fracture due to blunt thoracic trauma, hospitalized and treated in the department of General and Thoracic Surgery at the University Hospital "St. George" for the period between January 2010 and December 2012. The patients were included basing on the results from the imaging studies (conventional chest X-ray and computed tomography of the chest). For each patient we defined the sex, the age, the mechanism of chest injury, the number of fractured ribs, the type and the severity of associated intrathoracic injuries, hospital stay and the results from treatment.

\section{RESULTS}

The mean age of the patients was 53,9 years (from 15- to 91-years old). According to the age, the patients were divided in two groupspatients under the age of 65 years (147 cases, $69,3 \%)$, and patients over 65 years of age $(65$ patients, 20,7\%). The male patients were 161 , and the female patients were 51 . The analysis 
of the mechanism of trauma revealed that the leading cause for blunt chest trauma was the motor vehicle accident; the other factors are listed in Table 1. According to the number of fractured ribs, the patients were divided in two groups- group I included the patients with up to two fractured ribs (72 cases-33,9\%), and group II for the patients with multiple rib fractures ( $\leq 3$ ribs) - 140 patients $(66,1 \%)$. There were 8 cases of flail chest, all of them in patients under 65years of age. We found associated thoracic injuries in 36 of the cases from group I. The number of associated chest injuries was significantly higher in group II133 cases $(95 \%)$. The most common associated chest injury was the pulmonary contusion139patients $(65,6 \%)$, Figures 1 and 2 . Traumatic haemothorax was found in $21,2 \%$ of the cases, pneumothorax in $18,9 \%$ and haemopneumothorax in $11,8 \%$ of the cases. Fracture of the clavicle was diagnosed in 5,2\% of the patients, fracture of the sternum in $2,8 \%$
IV. DIMITROV, et al. of the cases, and fracture of the scapula in $2,4 \%$. The type of associated chest trauma and the distribution according the number of fractured ribs is presented in Table 2. The mean hospital stay was 8,7 days. Death occurred in 36 patients with rib fractures $(16,9 \%)$, the lethality rate was higher for the group of patients over the age of 65years. Death occurred up to the 72-th hour of admission in 13 patients $(6,1 \%)$, and after the 72 -th hour in 23 patients $(10,8 \%)$. The data revealed that the advanced age is a risk factor for mortality in patients underwent blunt chest trauma and rib fractures. Analyzing the correlation between the number of fractured ribs and the lethality rate, we found that increasing number or fractured ribs lead to higher mortality rate. The mortality rate was higher in patients with multiple fractured ribsthere was a positive correlation for the hole cohort, as for each of the groups divided by age.

Table 1. Mechanism of rib fractures, according the age distribution of the patients

\begin{tabular}{|lcccc|}
\hline \multicolumn{1}{|c}{$\begin{array}{c}\text { Mechanism of } \\
\text { trauma }\end{array}$} & $\begin{array}{c}\mathrm{N}=212 \\
(\%)\end{array}$ & $\begin{array}{c}\text { age } \leq 65 \\
(\%)\end{array}$ & $\begin{array}{c}\text { age }>65 \\
(\%)\end{array}$ & P \\
\hline 1.Motor vehicle accident & $121(57,1)$ & $94(63,9)$ & $27(41,5)$ & $<0,05$ \\
2. Pedestrian & $19(9,0)$ & $12(8,2)$ & $7(10,7)$ & - \\
3. Fall/Height/ & $42(19,8)$ & $27(18,4)$ & $15(23,1)$ & - \\
4. Fall/non-height/ & $13(6,1)$ & $1(0,7)$ & $12(18,5)$ & $<0,05$ \\
5. Other & $17(8,0)$ & $13(8,8)$ & $4(6,2)$ & - \\
\hline
\end{tabular}

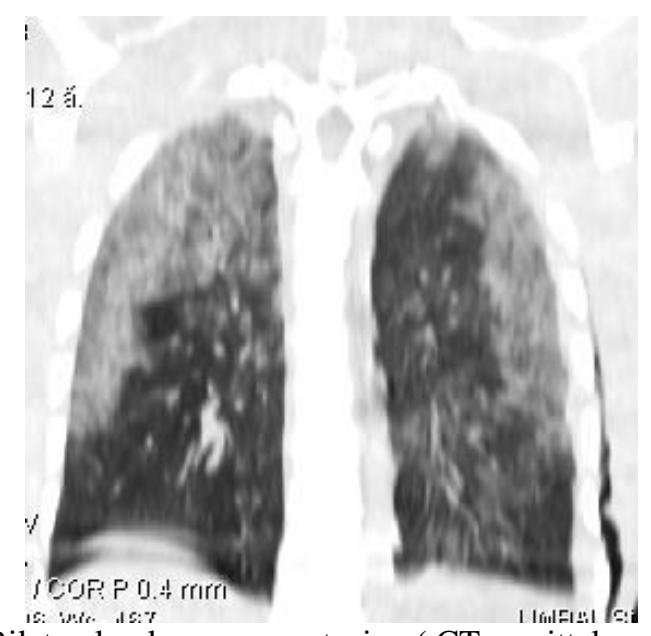

Figure 1. Bilateral pulmonary contusion ( CT- sagittal reconstruction)

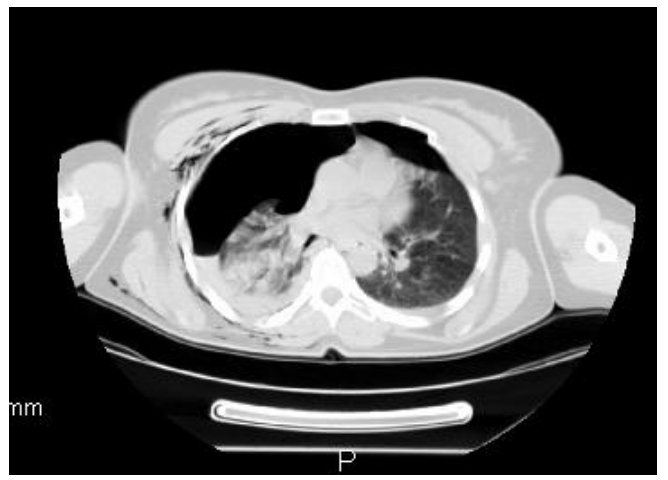

Figure 2. Bilateral rib fractures, right-sided pulmonary contusion and bilateral pneumothorax 
Table 2. Associated chest injuries, according the type of rib fracture

\begin{tabular}{|lcccc|}
\hline Associated chest injuries & $\mathbf{N}$ & $\begin{array}{c}\geq 2 \text { fractured } \\
\text { ribs }\end{array}$ & $\begin{array}{c}\text { Multiple } \\
\text { fractured } \\
\text { ribs( } \geq \mathbf{3} \text { ribs })\end{array}$ & p \\
\hline Pulmonary contusion & $\mathbf{1 3 9}$ & $\mathbf{1 5}$ & $\mathbf{1 2 4}$ & $<\mathbf{0 , 0 0 1}$ \\
Pulmonary lacerations & $\mathbf{3}$ & $\mathbf{0}$ & $\mathbf{3}$ & - \\
Pneumothorax & $\mathbf{4 0}$ & $\mathbf{1 0}$ & $\mathbf{3 0}$ & $<\mathbf{0 , 0 5}$ \\
Haemothorax & $\mathbf{4 5}$ & $\mathbf{5}$ & $\mathbf{4 0}$ & $\mathbf{0 , 0 5}$ \\
Haemopneumothorax & $\mathbf{2 5}$ & $\mathbf{3}$ & $\mathbf{2 2}$ & $<\mathbf{0 , 0 5}$ \\
Fracture of clavicle & $\mathbf{1 1}$ & $\mathbf{1}$ & $\mathbf{1 0}$ & $<\mathbf{0 , 0 0 1}$ \\
Sternal fracture & $\mathbf{6}$ & $\mathbf{2}$ & $\mathbf{4}$ & $<\mathbf{0 , 0 5}$ \\
Scapula fracture & $\mathbf{5}$ & $\mathbf{0}$ & $\mathbf{5}$ & - \\
\hline
\end{tabular}

Analyzing the lethality rate in patients underwent blunt chest trauma and rib fractures, we found associated thoracic injuries in all of cases ended with death. Therefore we concluded that the associated chest injuries are the immediate cause of death in these cases. In the cases ended with death up to the 72-th hour of admission, the autopsy protocols revealed that the cause of death was the pulmonary contusion. In three cases the cause of death was pulmonary contusion and haemorragic shock, as a result of lung lacerations and massive haemothorax. The data of imaging studies and autopsy protocols for the patients died after the 72-th hour of admission revealed that the cause of death is ARDS and post contusion pneumonia. We accept that both of these pathological conditions are a result and a complication of the pulmonary contusion.

\section{DISCUSSION}

Most of the thoracic traumas are caused by blunt trauma. Rib fractures are common, occurring in $10 \%$ to $26 \%$ of the cases $(10,11)$. Rib fractures are an indicator of considerable energy impact and frequently accompanied by various intrathoracic injuries. In adults the most frequent mechanism reported in the literature is the motor vehicle accident $(12,13$, 14). We report motor vehicle accidents as a cause of blunt thoracic trauma and rib fractures in $57,1 \%$ of the cases. In different studies of patients with rib fractures, the fraction of male patients was $60 \%$ to $70 \%$, predominantly younger population $(15,16)$. In our study the male patients are predominant, which is compatible with the cited data. The age of our patients varied between 15 and 91 years, and the group of patients under the age of 65 years included almost $70 \%$ of the cases. The age and sex distribution could be explained with the physical activity and the higher rate of motor vehicle use. The factors defining the severity of a blunt thoracic trauma with fractured ribs are the number of fractured ribs and the type and gravity of associated chest injuries. Our study demonstrated a positive correlation between the lethality rate and the number of fractured ribs. We found an increased mortality rate in patients with multiple rib fractures $(\geq 3$ ribs). Therefore we stated that the multiple rib fracture should be accepted as a risk factor in patients underwent blunt chest trauma.

The isolated rib fractures are not a serious clinical problem. The significance of the problem "rib fracture" is defined by the associated chest injuries. They are the ones responsible for the result of treatment in patients underwent blunt thoracic trauma and rib fracture; they are the immediate cause of death. There are many studies which showed positive correlation between the number of fractured ribs and the gravity of associated intrathoracic injuries $(17,18)$. Our study showed higher rate of associated chest injuries in patients with multiple rib fractures. The pulmonary contusion was the most encountered one- $65,6 \%$ of the cases. This frequency was compatible with the cited one in the literature. There is data of many experimental and clinical studies, which showed that the pulmonary contusion and its complications are the most common cause of death in patients underwent blunt chest trauma $(19,20)$. Our results showed that all of cases ended with death are due to pulmonary contusion and/or its complications-ARDS and post contusion pneumonia. Pulmonary contusion and its complications is considered as the most common cause of death in hospitalized blunt thoracic trauma patients. The mortality rate in our study is compatible with one cited in the literature.

\section{CONCLUSIONS}

Our retrospective study presented the patterns of associated thoracic injuries in patients underwent blunt chest trauma and rib fractures. Our results confirmed the role of rib fractures as a marker of severity in blunt chest trauma and showed that the immediate cause of death in these cases is the associated chest injury, mostly pulmonary contusion. 


\section{REFERENCES}

1. Cameron J. Current Surgical Therapy. 8th ed. St. Louis, MO: Mosby; 2004.

2. Shorr RM, Crittenden M, Indeck M, et al. Bluntthoracic trauma. Analysis of 515 patients. Ann Surg; 206:200-5, 1987.

3. Greene R. Lung alterations in thoracic trauma. J Thorac Imaging; 2:1-11, 1987.

4. Holcomb JB, McMullin NR, Kozar RA, Lygas MH, MooreFA.Morbidity from rib fractures increases after age 45. JAm Coll Surg;196:549-55, 2003.

5. Karadayi S, Nadir A, Sahin E, Celik B, Arslan S, KaptanogluM. An analysis of 214 cases of rib fractures. Clinics(Sao Paulo); 66:449-51, 2011.

6. Yucel O, Sapmaz E, Caylak H, et al. Hastaneye yatirilmayigerektiren toraks travmali 748 olgunun analizi. Gulhane TipDergisi; 51:86-90, 2009.

7. Flagel BT, Luchette FA, Reed RL, et al. Half-a-dozen ribs:the breakpoint for mortality. Surgery;138:717-23, 2005.

8. Bergeron E, Lavoie A, Clas D, et al. Elderly trauma patientswith rib fractures are at greater risk of death and pneumonia. $J$ Trauma;54:478-85, 2003.

9. Easter A. Management of patients with multiple rib fractures. Am J Crit Care; 10:320-27, 2001.

10.Ziegler DW, Agarwal NM. The morbidity and mortality of rib fractures.J Trauma; 37:975-9, 1994.

11.Liman ST, Kuzucu A, Tastepe AL, et al. Chest injury due to blunttrauma. Eur $J$ Cardiothorac Surg; 24:374-8, 2003.
IV. DIMITROV, et al.

12.Flagel BT, Luchette FA, Reed RL, et al. Half-a-dozen ribs: the breakpointfor mortality. Surgery; 138:723-5, 2005.

13. Sirmali $M$, Turut $H$, Topcu $S$, et al. A comprehensive analysis of traumaticrib fractures: morbidity, mortality and management. Eur $J$ CardiothoracSurg; 24:133-8, 2003.

14.Bergeron E, Lavoie A, Clas D, et al. Elderly trauma patients with ribfractures are at greater risk of death and pneumonia. $J$ Trauma; 54:478-85, 2003.

15.Sirmali M, Turut H, Topcu S, Gulhan E, Yazici U, Kaya S, et al. Acomprehensive analysis of traumatic rib fractures: morbidity, mortalityand management.Eur $J$ Cardiothorac Surg.; 24:133-8, 2003.

16. Testerman GM. Adverse Outcomes in Younger Rib Fracture Patients.Southern Medical Journal. Volume 99, Number 4, April 2006.

17.Flagel BT. Half-a-dozen ribs: The breakpoint for mortality. Surgery; 138: 717725, 2005.

18. Hassani AA et al. Rib fracture patterns predict thoracic chest wall and abdominal solid organ injury. The Am. Surg; 8:888891, 2010.

19.National Trauma Data Bank (NTDB_). American Collegeof Surgeons. Available at: http://www.facs.org/trauma/ntdb.html. Accessed: June 2003.

20.Croce MA, Tolley EA, Fabian TCA: formulafor prediction of posttraumatic pneumoniabased on early anatomic and physiologic parameters. J Trauma; 54:724730, 2003. 